\title{
INDAGAÇÃO DIALÓGICA DE GORDON WELLS EM PROCESSOS FORMATIVOS COM PROFESSORES: Interlocuções com a Comunidade Científica Internacional
}

\author{
Anahy Arrieche Fazio ${ }^{1}$ \\ Valmir Heckler ${ }^{2}$ \\ Maria do Carmo Galiazzi $i^{3}$
}

\begin{abstract}
RESUMO
O estudo comunica, em uma perspectiva fenomenológica hermenêutica, o que se mostra da indagação dialógica de Gordon Wells em comunicações da comunidade científica internacional que envolvam processos formativos docentes. Desenvolveu-se uma pesquisa exploratório-descritiva a partir das 10 obras mais citadas do autor na base de dados do Google Acadêmico. Foram selecionados 119 trabalhos. Estes foram lidos integralmente e organizados em sínteses traduzidas para a língua portuguesa, evidenciando os aspectos teórico-práticos da indagação dialógica em cada um deles. Emergiram do processo da análise cinco categorias: i) Escrita, Leitura e Comunicação via Ferramentas Tecnológicas; ii) A Interatividade e a Colaboração em Redes; iii) Desenvolvimento Profissional em Comunidades; iv) Aprendizagem Colaborativa no Ensino de Ciências; v) Tutoria e Mediação no Desenvolvimento Profissional. Os resultados indicam que os aspectos que se mostram em relação aos contextos em que a indagação dialógica de Gordon Wells é integrada fornecem pistas para que seja possível compreender o desenvolvimento profissional em um viés dialógico, social e colaborativo em ambientes com sujeitos diferentes.

Palavras-chave: Gordon Wells; indagação dialógica; desenvolvimento profissional; processo formativo.
\end{abstract}

\section{GORDON WELLS' DIALOGICAL INQUIRY IN FORMATIVE PROCESSES WITH TEACHERS:} INTERLOCUTIONS WITH THE INTERNATIONAL SCIENTIFIC COMMUNITY

\begin{abstract}
The study communicates, in a hermeneutic phenomenological perspective, what is it, Gordon Wells' dialogic inquiry in communications from the international scientific community that involve formative processes, like. An exploratory-descriptive research was developed from the 10 most cited works by the author in the Google Scholar database. The works selected, 119, were completely read and organized into summaries, translated into Portuguese, highlighting the theoretical-practical aspects of the dialogic inquiry in each of them. From the analysis process five categories emerged: i) Writing, Reading and Communication via Technological Tools; ii) Interactivity and Collaboration in Networks; iii) Professional Development in Communities; iv) Collaborative Learning in Science Teaching; v) Tutoring and Mediation in Professional Development. The emerging aspects, related to the contexts in which Gordon Wells' dialogic inquiry is integrated, provide clues to understand professional development in a dialogic, social and collaborative perspective in environments with different subjects Keywords: Gordon Wells; dialogical inquiry; professional development; formative process.
\end{abstract}

RECEBIDO EM: 30/8/2021

ACEITO EM: 4/11/2021

\footnotetext{
${ }^{1}$ Autora correspondente. Universidade Federal do Rio Grande - Furg. Av. Itália, s/n - km 8, Carreiros. Rio Grande/RS, Brasil. http://lattes. cnpq.br/8514050485623854. https://orcid.org/0000-0003-4067-7983.anahyfazio@yahoo.com.br

2 Universidade Federal do Rio Grande - Furg. Rio Grande/RS, Brasil. http://lattes.cnpq.br/0446681267010261. https://orcid.org/0000-00023838-3903.

3 Universidade Federal do Rio Grande - Furg. Rio Grande/RS, Brasil. http://lattes.cnpq.br/4430976902171474. https://orcid.org/0000-00030513-0018.
} 


\section{INTRODUÇÃO}

O artigo comunica compreensões acerca da indagação dialógica de Gordon Wells, em uma perspectiva fenomenológica-hermenêutica (BICUDO, 2011), contextualizada em processos formativos com professores a partir da interlocução com a comunidade científica internacional. $O$ questionamento central do estudo é: $O$ que se mostra da indagação dialógica de Gordon Wells em comunicações da comunidade científica internacional que abordam processos formativos? Transformation foi a palavra escolhida por Gordon Wells (2001) para caracterizar a principal proposta da educação, em que todos os envolvidos transformam suas formas de agir, pensar, sentir, em que o professor tem relevante papel ao criar oportunidades de transformação pessoal e social dos envolvidos.

No campo da formação de professores, tema central deste estudo, Gordon Wells (1999) propõe o desenvolvimento de comunidades colaborativas na perspectiva da indagação dialógica, em que os processos de ensino e de aprendizagem acontecem no indagar, em que o professor busca uma sala de aula pautada na argumentação e no questionamento, compreendendo a investigação como um processo coletivo (WELLS, 2016 ). A ideia de comunidades dialógicas é amplamente discutida no livro Dialogic Inquiry (1999), em que o autor contextualiza a criação de um grupo de formação de professores constituído por atuantes nas escolas e na universidade. O referido autor assume ser a formação de comunidade de professores um processo formativo horizontal, uma vez que à medida que os professores se engajam em atividades coletivas, transformam suas práticas e metodologias, ao mesmo tempo que transformam sua relação com a comunidade e na comunidade (WELLS, 2001).

Moraes (2011), a partir de Wells, apresenta essas comunidades para um contexto virtual, entendendo que os espaços virtuais e sua diversidade de ferramentas e recursos multiplicam possibilidades de conversas e interação entre estudantes, tutores e professores, em que cada sujeito revisita suas aprendizagens de forma dialógica. Esses pressupostos de uma abordagem sociocultural de educação dialógica fomentam a formação de professores de Ciências da Universidade Federal do Rio Grande (Furg) e são expressos no curso de Licenciatura em Ciências EaD, campo de atuação dos autores deste artigo, em que a formação de professores de Ciências foi planejada e desenvolvida no contexto de comunidades dialógicas a partir de Gordon Wells.

Para Wells (1999), a formação de professores está imersa em teorias socioculturais de aprendizagem e na promoção de ações investigativas durante sua prática. Os professores colocam-se na posição de aprendentes, em que são encorajados e responsabilizados pelo seu aprendizado. Aprendem uns com os outros enquanto se engajam em processos de indagação dialógica. Nesse contexto, a indagação tem suas raízes no aspecto histórico, nas compreensões do passado, e no cultural, ao incorporar compreensões do passado às práticas e aos artefatos culturais. No contexto da sala de aula, a prática da indagação orienta para a construção de compreensões teórico-práticas (WELLS 1999, 2001, 2002).

O contexto social e a interação são ideias defendidas por Paula e Harres (2016), em que compreendem que as propostas didáticas devem ser organizadas de forma a promover a interação social como condição para a aprendizagem, tomando os princí- 
pios do educar pela pesquisa, perguntar, argumentar, debater, como uma proposta que vai ao encontro desse objetivo. Wells (1999) observa que, no contexto de uma aula de Ciências, as crianças trabalham coletivamente para encontrar soluções aos problemas propostos, construindo modelos a partir dos desafios sugeridos pelo professor. Desse estudo, acrescenta que embora tenham aprendido princípios básicos de eletricidade, a socialização com a comunidade, em feiras de ciências, workshops, mesas-redondas, entre outros, dos modelos construídos, seguindo a abordagem orientada à indagação, criou oportunidades para que os estudantes se engajassem em muitas formas de discurso, tanto falado quanto escrito.

Compreensão similar é significada por Guidotti e Heckler (2021) em um contexto da Educação em Ciências. Os autores entendem que a aprendizagem por meio da investigação acontece com o diálogo entre os sujeitos como estratégia didático-pedagógica, que desafia o professor a escutar, problematizar e contextualizar saberes e vivenciar novas experiências.

Kenski, Medeiros e Ordéas (2019) argumentam que as tecnologias digitais redimensionam os papéis dos estudantes e dos professores, no contexto da Educação Superior, modificando a lógica do ensino centrado no professor. Os autores, portanto, apontam que essa nova postura demanda a readequação de algumas medidas básicas que as instituições de ensino precisam reorganizar, entre elas,o movimento de repensar a formação de professores, inicial e continuada, com vista à utilização das tecnologias digitais nos processos educativos e o estímulo ao senso colaborativo e interativo on-line "de forma a que a presença virtual seja mais importante e significativa do que a presença passiva dos estudantes em salas de aula presenciais" (2019, p. 11).

Considerando o contexto da formação de professores em um viés dialógico, seguindo as ideias de Wells e as experiências registradas pelos autores supracitados no referido campo, emerge a necessidade de compreender como a indagação dialógica vem suscitando transformações no campo da formação de professores ao redor do mundo. Como as ideias de Wells são contextualizadas nas diversas experiências formativas comunicadas na comunidade científica internacional? Quais os contextos em que essas são desenvolvidas e como são organizadas? Como os diferentes artefatos e interfaces são utilizados neste contexto? Que aspectos teórico-práticos da formação são comunicados? Partindo dessas indagações, objetiva-se compreender o que se mostra da indagação dialógica, na perspectiva de Gordon Wells, na interlocução com diferentes pesquisadores a partir de suas publicações científicas.

\section{METODOLOGIA}

Com o objetivo de compreender o que se mostra das ideias de Wells comunicadas em estudos sobre sua instituição em diferentes contextos, este trabalho configura-se como uma pesquisa exploratória-descritiva (VERGARA; PECl, 2003) de natureza fenomenológica-hermenêutica (BICUDO, 2011).

Nesse viés, a compreensão do fenômeno envolve o olhar ao contexto das experiências vividas, buscando modos e sujeitos significativos que possam dizer de suas experiências de maneira que vá ao encontro da interrogação e permita a construção de significados sobre o fenômeno a ser compreendido (BICUDO, 2011). Nessa busca, as- 
sume-se uma perspectiva exploratória, investigando diferentes interlocutores que referem assumir Gordon Wells como fundamento em seus processos formativos comunicados, buscando compreensões acerca do campo e da temática de estudo que, em um movimento dialógico, possibilitará novas compreensões sobre o fenômeno investigado a partir dos registros das experiências de trabalhos de outros pesquisadores.

Como fonte da pesquisa na literatura, optou-se pela plataforma de pesquisa Google Acadêmico, considerando sua cobertura que inclui uma grande quantidade de documentos (MARTIN-MARTIN et al., 2020). O Google Acadêmico apresenta a possibilidade do Perfil do Usuário, em que apresenta as estatísticas de citações, dados acadêmicos do pesquisador e as redes de conversação. A partir do perfil do pesquisador Gordon Wells é possível ordenar a listagem de obras pela ordem alfabética dos títulos, por ordem crescente ou decrescente de citações ou ano. No movimento de investigar os contextos em que o pesquisador é citado, ordenamos as obras pelo número de citações e nos concentramos nas 10 obras mais citadas do autor, optando pela exclusão de uma das obras focada na alfabetização de crianças, considerando o afastamento do contexto desta pesquisa. Os títulos escolhidos e o seu ano de produção estão indicados no Quadro 1.

Quadro 1 - Relação de obras mais citadas de Gordon Wells

\begin{tabular}{|c|l|c|}
\hline \multicolumn{2}{|c|}{ Título } & Ano \\
\hline 1 & Dialogic inquiry: Towards a socio-cultural practice and theory of education & 1999 \\
\hline 2 & The meaning makers: Learning to Talk and Talking to Learn & 2009 \\
\hline 3 & Learning through interaction: volume I: the study of language development & 1981 \\
\hline 4 & Dialogic inquiry in education: building in the Legacy of Vygotshy & 2000 \\
\hline 5 & What's the use of 'triadic dialogue'?: An investigation of teacher-student interaction & 2000 \\
\hline 6 & Learning for life in the 2lst century: Sociocultural perspectives on the future of education & 2002 \\
\hline 7 & Action, talk, and text: Learning and teaching through inquiry & 2001 \\
\hline 8 & Changing schools from within: Creating communities of inquiry & 1993 \\
\hline 9 & Language and the inquiry-oriented curriculum & 1995 \\
\hline 10 & $\begin{array}{l}\text { Language, learning, and education: Selected papers from the Bristol study, Language at } \\
\text { Home and School }\end{array}$ & 1985 \\
\hline
\end{tabular}

Fonte: Os autores (2021).

Na coluna ao lado dos títulos, no Google Acadêmico, os números indicam os trabalhos que citam essas obras na sua escrita e ao clicar no total das citações para cada obra é possível visualizar a listagem das obras em que as citações foram encontradas. A partir dessa listagem, realizou-se a busca específica dentre as obras por meio da opção "Pesquisar nos artigos de citação". Essa etapa é importante para o refinamento das informações, permitindo que o usuário aponte palavras-chave ou termos específicos que deseja buscar nos trabalhos. 
Com o intuito de buscar consonância com o contexto de desenvolvimento dos estudos deste artigo, fez-se a escolha de descritores, em inglês, e foi realizada a busca de todas essas palavras, uma por vez, entre as citações das dez obras escolhidas. Os descritores escolhidos foram: "teacher formation", "community development", "on-line community", "wenger", "on-line science teacher", "community of practice"; "distance education", "virtual learning environment", "learning management system" e "learning community". Para a pesquisa do descritor de forma literal, do termo como um todo, as aspas foram colocadas. A busca pelos descritores ocorreu em maio de 2019 , individualmente em cada uma das dez obras e resultou em 10.429 produções. Em seguida, buscou-se maior aproximação ao campo e objeto da pesquisa, os descritores foram combinados em grupos de descritores, cada um entre aspas e separados por ";" para que os trabaIhos emergentes apresentassem os termos simultaneamente. Desta etapa, registrou-se o quantitativo de 288 trabalhos, os quais, em um primeiro momento, foram reduzidos a 143 obras, após a exclusão das que foram contabilizadas em mais de um grupo de descritores e/ou documentos não referenciados ou contextualizados ao campo de estudo.

Em um segundo momento, o olhar voltou-se ao conteúdo dessas produções em um movimento descritivo-interpretativo na perspectiva fenomenológico-hermenêutica (BICUDO, 2011) a partir da tradução das obras, ${ }^{4}$ organização das informações e sistematização de compreensões em categorias emergentes.

As produções que não estavam na íntegra e a revisão de livro foram descartadas da análise, que ao fim, totalizou 119 produções, cujas referências estão disponíveis no Apêndice. Observa-se nos registros bibliométricos aspectos relativos à natureza das obras e anos de publicação. Quanto à natureza dessas obras, percebe-se que a maioria das produções são teses de Doutorado, seguidas de artigos e capítulos de livro e outras formas de produção, como pode ser visualizado na Figura 1. Registra-se que a maioria das produções ocorreram em 2013, congruente com o ano em que ocorreram mais citações das obras de Wells, segundo seu perfil no Google Acadêmico.

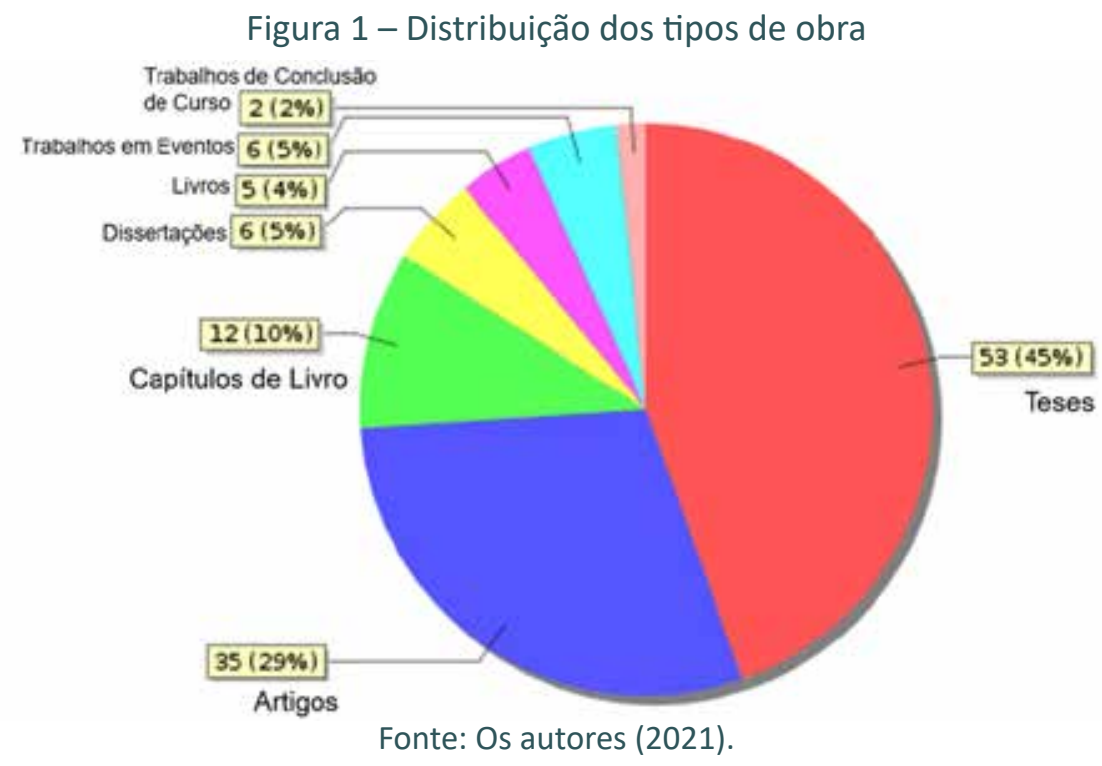

${ }^{4}$ Os autores se responsabilizam pela tradução das obras 
O processo descritivo é iniciado pela leitura hermenêutica, interpretando e transportando as experiências em linguagem escrita. Nessa comunicação, ainda se apresenta o processo natural de significação da linguagem, uma vez que os textos estudados não foram comunicados no idioma em que este artigo está sendo desenvolvido. Reitera-se, em Bicudo (2011, p. 44), que:

[...] toda a análise de descrição, por ser mediada na linguagem, solicita um enxerto hermenêutico, para que no movimento de compreensão do dito, já se proceda à abertura de sentidos e significados expressos e transportados pelo modo de dizer pelo qual a descrição se doa à interpretação (p. 44).

Nesse movimento hermenêutico as 119 produções foram lidas e sintetizadas, em Português, de forma a elucidar os objetivos, o contexto em que Wells é citado, os resultados e as conclusões desses trabalhos. Da leitura dessas sínteses foram destacadas palavras que contribuem para a aproximação entre esses interlocutores, e extraiu-se, então, excertos de cada síntese que foram organizadas em sentenças que respondem à interrogação formulada: $O$ que se mostra da indagação dialógica de Gordon Wells em publicações científicas? A partir das ideias comunicadas, os trabalhos foram agrupados e classificados em cinco categorias: i) Escrita, Leitura e Comunicação via Ferramentas Tecnológicas; ii) A Interatividade e a Colaboração em Redes; iii) Desenvolvimento Profissional em Comunidades; iv) Aprendizagem Colaborativa no Ensino de Ciências; v) Tutoria e Mediação no Desenvolvimento Profissional. As principais ideias entre essas categorias serão comunicadas na sequência.

\section{RESULTADOS E DISCUSSÃO}

\section{Escrita, Leitura e Comunicação via Ferramentas Tecnológicas}

Nesta categoria emergem os trabalhos que abordam o uso das ferramentas tecnológicas na escrita e leitura em processos de aprendizagem. Mediante as dificuldades em se desenvolver a escrita colaborativa (REZEKI, 2016), as ferramentas tecnológicas oferecem possibilidade de leitura, releitura, incentivo a escrita, avaliação por pares (HUANG, 2012) e a coconstrução do conhecimento, que abrange o afeto e a interação (YUKAWA, 2005; YEN, 2011), considerando ainda o desenvolvimento de valores e habilidades sociais enquanto se escreve com pares (REZEKI, 2016). Nesse viés, as ferramentas tecnológicas auxiliam a melhorar a escrita e a leitura nos processos de autoria, produção e consumo nas salas de aula (PAÉZ, 2015) por meio da promoção de atividades intertextuais, conectadas a textos, fotos, livros, multimídia e outros recursos no multiletramento (BEZAIRE, 2009).

A escrita dialógica, monológica e colaborativa acontece em Ambiente Virtual de Aprendizado (AVA), wiki, chat, web, painel eletrônico, blog e rede social, e é promotora do aprendizado em redes (MATTHEW; FELVEGI; CALLAWAY, 2014; HUANG, 2012; BARTLET-BRAGG, 2013; SPILIOTOPOULOS; CAREY, 2016). Em fóruns, a escrita argumentativa acontece mediante a proposição de desafios (FLYNN; POLIN, 2003). Significa que o uso de um ambiente virtual de aprendizagem em rede social oferece riqueza e liberdade para criar, anotar, avaliar e comentar sobre diferentes artefatos (HILL, 2011; SHUKOR, 2014; GONZÁLEZ, 2015; BERRY, 2014), considerando que aspectos físicos e sociais do ambiente da sala de aula influenciam na criação de condições para a atividade (BEZAIRE, 2009). 
Nesse âmbito também são sugeridos software colaborativos baseados em aprendizagem investigativa (YUKAWA, 2005) e o uso de ambientes de aprendizagem colaborativos personalizados (FARMER; BARTLET-BRAGG, 2005), em que se reitera a importância do professor como facilitador dos processos ao propor ferramentas e recursos que envolvam os estudantes em novas formas de comunicação e interação (PÁEZ, 2015).

\section{A Interatividade e a Colaboração em Redes}

Nesta categoria de análise mostra-se que as ferramentas de comunicação síncrona e assíncrona baseadas na Internet e mediadas por computador (CMC) (fórum, e-mail, chat síncrono) são muito importantes para proporcionar interação e colaboração em comunidades on-line (KHOO, 2010; MARDEN, 2016; LEE, 2005; CHAI; TAN, 2006; TAN, 2018, 2019). Por exemplo, o uso de ferramentas de diálogo aumenta a participação de estudantes em discursos acadêmicos e provê oportunidades para interações críticas (YIM, 2011; MORGAN, 2015).

A conversação, as dinâmicas de observação e reflexão a e construção de espaços mentais colaborativos em uma comunidade virtual possibilitam a presença social, o sentimento de pertencimento, identidade, o desenvolvimento da prática de ensino compartilhada, contato transcultural e o pensar reflexivo a partir da contribuição com outros sujeitos (GRAETZ, 2008; KELLY et al., 2016; HELLERMANN, 2008; CHRISTIANSEN, 2010). Os modelos interativos são um esforço de sobrepor o download mecânico de textos no ensino a distância (BAKER; WATSON, 2014), contudo neste modelo é preciso enfatizar o papel crucial dos fatores sociais e históricos na forma de organizar a participação (WESTBERRY, 2009).

Na participação em discussões os cursistas repensam/apresentam pontos de vista e criam novas compreensões e significados, em que o diálogo colaborativo reflexivo evoca diferentes maneiras de saber, inspira autenticidade e melhora o aprendizado (SARITAS, 2006; VICKERS-MANZIN; JOHNSTONS, 2013). Nisso, o desenvolvimento profissional do professor é essencial na promoção de aulas interativas e dialógicas. As tecnologias educacionais dão suporte para que os professores em formação mudem seu comportamento no grupo e isso abrange a transformação de atitudes, crenças e valores a respeito dos conhecimentos pedagógicos e tecnológicos adquiridos (DONNELLY, 2009; HENNESSY; LONDON, 2013; KELLY, 2019).

A transformação envolve projetar um ambiente de aprendizado que esclareça sobre os processos de discussão on-line. $O$ ambiente necessita evidenciar os objetivos e as instruções de participação com os conteúdos, e encorajar estudantes a compartilharem opiniões e divergências na busca por promover a interatividade com e entre os diferentes estudantes (SARITAS, 2006; SIGALA, 2007; LOCKHORST, 2004; HAN, 2013). Para isso, as tarefas devem ser estruturadas de maneira a estimular o envolvimento entre os alunos e destes com os conteúdos, por meio de fóruns e feedbacks (BAKER; WATSON, 2014; JAMIL, 2015; STEELE, 2009).

Para além da estruturação do ambiente de aprendizagem, é preciso consonância dos aspectos metodológicos com as teorias de aprendizagem para que incentivem a negociação entre os participantes (LEE, 2009). Assim, o uso de projetos, apresentações, investigações, mecanismos proativos de resolução de problemas, abordagens em an- 
daimes ${ }^{5}$ são possibilidades no incentivar a interação, a discussão e a coconstrução entre estudantes, sempre com o auxílio do professor, contribuindo para o desenvolvimento profissional, a transformação educacional e a autonomia no aprendizado (BUNCH, 2013; DONNELLY, 2009; GRAETZ, 2007; RESCHKE, 2001; KLENNER-MOORE, 2002; LUND; HAUGE, 2011; MERI-YILAN, 2017). Nisso também se coloca a importância da mediação do professor/instrutor, seu carinho e receptividade, ao reconhecer o papel da experiência e a participação de estudantes mais experientes, da cultura dos indivíduos e das atividades sociais que promovam a interação dialética (YIM, 2011; CHILDS, 2011; GELER; NEISLER, 2014; PRITCHARD, 2010; STONE, 2018; SIMPSON, 2016; HAUGHT, 2005; MAGGIOLI, 2018; FAHLE, 2019), uma vez que a falta de mediação pode contribuir para a não efetividade das ferramentas como, por exemplo, do fórum na formação de professores (MCPHEE, 2013).

Nesse contexto, professores e estudantes constroem significados e trocam ideias em um verdadeiro coaprendizado. Trata-se de uma forma de ampliar compreensões teórico-práticas acerca do ensino dialógico, em que cada indivíduo é influenciado e sustentado pela atividade e o pensar dos outros (JAWORSKI, 2014; HENNESSY; MERCER; WARWICK, 2011). A produção acadêmica considera que todo tipo de indagação resulta na coconstrução de conhecimentos, portanto é importante para o desenvolvimento profissional a colaboração entre professores com diferentes experiências e níveis educacionais (JAWORSKI, 2014), em prol de uma jornada formativa que é construída a partir da reflexão e ressignificação das experiências dos professores, para além da ação (MCPHEE, 2013). Assim, emerge o desafio de dialogar sobre as comunidades que buscam o desenvolvimento profissional.

\section{Desenvolvimento Profissional em Comunidades}

Nesta categoria emergem aspectos de comunidades que colaboram para o desenvolvimento profissional a partir da transformação de crenças teórico-práticas dos participantes. Mostra-se que a adoção de tecnologias na educação depende do rompimento da fronteira entre a cultura acadêmica e a social, fazendo-se necessário reconsiderar a linguagem utilizada com exposição a práticas de discurso, uma vez que sem mudar os paradigmas educacionais as ferramentas são apenas "pedaços de tecnologia" (CARON; CARONIA, 2008; THOMPSON, 2008; SINCLAIR, 2004). Com o uso da tecnologia, criam-se oportunidades de (re)contextualizar o aprendizado em ambientes de trabalho, considerando a experiência como fundamental na construção de sentidos sobre a prática acadêmica, uma vez que o desenvolvimento profissional colaborativo atrelado à tecnologia é potencializador do aprendizado e de oportunidades para a formação continuada (THOMPSON, 2008; ARTEKIN, 2013; BERNASCONI, 2010; JEWITT, 2018; DOYLE, 2014; TAPP, 2013).

Quanto aos recursos, do uso das tecnologias, aplicativos móveis, televisão, vídeos, leituras on-line, entre outros, cria-se a necessidade de uma visão transformadora nas salas de aula. Exige-se um movimento de descentralização da figura do professor e de

No contexto da sala de aula é um processo de interação entre sujeitos em que o professor - ou um sujeito mais experiente - presta assistência e guia os estudantes na construção do conhecimento. 
imersão em diferentes contextos, encorajando os futuros professores a reinterpretarem seu conhecimento, valores e crenças que possam impactar na sua prática profissional (BAINBRIDGE et al., 2013; GAUDIN; CHALIÈS, 2015; SEELS; FULLERTON; BARRY, 2008; COONER, 2010) . O uso de vídeos na educação de professores, em programas profissionais, constituem importantes ferramentas para a aprendizagem e, ao mesmo tempo, para que o professor analise sua própria prática e, quando associadas à web, propiciam a construção da identidade profissional de professores, ao compreenderem a reflexão como prática social e individual (GAUDIN; CHALIÈS, 2015; HUNG, 2005).

Nessa perspectiva, o uso das tecnologias de informação e comunicação em contextos educacionais propiciam a comunicação, a conversação, o desenvolvimento de habilidades de colaboração em comunidades de aprendizagem (TWINING et al., 2006; ROBUTTI et al., 2016). Nisso, mostra-se que o uso de tecnologias atreladas a ambientes de aprendizagem híbridos é uma combinação propícia para a realização de atividades conjuntas de solução de problemas. É importante destacar, no entanto, que as dificuldades apresentadas pelos professores em relação ao ensino híbrido provêm dos padrões pedagógicos tradicionais, da fragmentação entre os conteúdos tecnológicos, pedagógicos e conceituais (ENOCH, 2016; HOANG, 2015), da falta de processos logísticos básicos e de uma política de e-learning ${ }^{6}$ que dificultam a adoção de tecnologias educacionais em programas acadêmicos (MLITWA, 2017).

As comunidades de aprendizagem on-line são importantes para que os professores possam buscar aporte tecnológico e pedagógico, vivenciando transições em que seus modelos culturais são desafiados, revelando novas abordagens metodológicas que enfatizam a importância do planejamento, reflexão e ensino por investigação (BRUCE; SULLIVAN, 2018; AWENOWICKS, 2009; HADDOCK, 2014; HILL et al., 2008). O engajamento dos participantes e a presença na comunidade dependerá da motivação e acompanhamento do educador na universidade (FARROQ, 2019). Nas comunidades de indagação uma ferramenta importante é fornecer feedback frequente aos participantes e aos que estruturam os cursos e/ou programas, em uma perspectiva do modelo de colaboração para a construção de ambientes de aprendizagem eficazes, reconhecendo o papel das tecnologias como ferramentas cognitivas no ensino-aprendizagem. (CHAI, 2006; MOREIRA; ALMEIDA, 2011).

Observa-se que nas comunidades colaborativas o desenvolvimento profissional acontece pela partilha de interesses de profissionais que evoluem socialmente e individualmente (KHOUSA; ATHIF; MASUD, 2015), uma vez que emergem necessidades e interesses comuns em um ambiente de confiança mútua. Isso acontece com a inserção dos estudantes em práticas com diferentes profissionais, oportunidades em que assim aprendem a fazer parte de uma comunidade de prática (FARREL; BADKA, 2015; QUIÑONES QUIÑONES, 2017). Nisso se apresenta a educação baseada na prática como uma metodologia que prepara os graduandos para suas futuras ocupações práticas, na melhora das habilidades de liderança e colaboração, inclusive estando estes geograficamente distantes (CLARKE, 2017). Abrange um envolver os participantes a pensar so-

Eletronic Learning ou e-learning é uma terminologia utilizada para se referir às diversas abordagens de aprendizagem com suporte de tecnologia (JAMIL, 2015) 
bre papéis e identidades que perpassam suas futuras ocupações, engajando-os em uma práxis emocional crítica com enfoque em aspectos emocionais do ensinar, para trabaIhar por mudanças pessoais, profissionais, sociais e institucionais (SHEEHAN; HIGGS, 2013; CLARKE, 2008; PADILLA, 2014). Assim, registra-se que as TICs nas comunidades com desenvolvimento profissional oportunizam mudanças pessoais, profissionais, sociais e institucionais.

\section{Aprendizagem Colaborativa no Ensino de Ciências}

Incluem-se nesta categoria os interlocutores que argumentam sobre o contexto das comunidades atreladas às Ciências. Considera-se o ambiente bilíngue da ciência que pode incitar dificuldades de aprendizagem -- e é um fenômeno instrucional multifacetado negociado por intermédio de interações multilaterais e abordagens que possibilitam a interação professor-estudante e estudante-estudante (DESTINO, 1994).

Para tal, é necessário encorajar os processos discursivos por meio da indagação, investigação, uso de modelos animados, resolução de problemas, projetos, apresentações e registros escritos (fóruns e cadernos), que estimulam os estudantes a falarem sobre os fenômenos científicos, a reconhecerem fenômenos que não são empiricamente observáveis e usá-los, propiciando aprendizado e construção de entendimentos de Ciências (QHOBELA, 2008; CHEN, 2006; KARLSSON, 2010; WILMES, 2017). Neste viés, ressalta-se a importância da escrita como facilitadora do movimento entre discursos, incitando o uso e compreensão da linguagem científica, e mostrando a variedade de formas em que os significados são negociados pelo uso da linguagem (ANSON, 2017; ROWELL, 2008; ZULU, 2018).

O papel do docente é uma complexa mistura de aprendente, ousado, indagador, desenvolvedor de currículo, negociador, colaborador e professor (SLAVIT; NELSON; LESSEIG, 2016). O professor gerencia a interação e propicia suporte à indagação coletiva, estimulando os estudantes a criarem hipóteses, questionarem, compartilharem experiências diárias, ouvindo, respeitando e retroalimentando seus colegas em uma perspectiva social, cognitiva e socioemocional (KOVALAINEN, 2013).

Por meio do desenvolvimento colaborativo profissional e das comunidades de prática os professores aprendem novos pressupostos teóricos, desenvolvem e revisitam o currículo, as práticas pedagógicas e de linguagem científica, dialogam sobre fatores estressores e aproximam sua prática de suas crenças, confrontam e refletem sobre aspectos teórico-práticos em relação ao seu contexto cultural, dentro de sua comunidade de prática. (SUBRAMANIAN, 2001; NELSON, 2009; TROIANO, 2012; VERJOVISKY; WALDEGG, 2005; COPPERSMITH, 2017). Não obstante, professores pesquisam sua própria prática em colaboração profissional com outros docentes e envolvidos em atividades colaborativas, que incentivam a produção de materiais, bem como a análise de seus discursos, conduzindo à transformação do repertório desses discursos nas suas aulas de Ciências e possibilitam a reflexão crítica na construção de significados (NELSON, 2009; KOKKOTAS; PILIOURAS, 2005; KOKKOTAS et al., 2009; PILIOURAS; PLAKITSI; NASIS, 2015; PLAKITSI; PILIOURAS; EFTHIMIOU, 2017). 
É preciso, portanto, desenvolver ferramentas que deem suporte para os usuários reaprenderem o que eles já fazem, porém de uma maneira melhor, superando o uso da tecnologia como forma de cobrir o conteúdo e propiciando um ambiente que encoraje as discussões e interações (BARAB; EVANS; BACK, 2009; CHEN, 2006). Para tal, conclui-se que o desenvolvimento de cursos que expandam o horizonte sobre as práticas educacionais por meio de comunidades em que os participantes desenvolvam abordagens de liderança, e que essas experiências entre professores, coletivamente, façam parte de um movimento de melhoria do Ensino de Ciências, é uma via para a transformação (NELSON, 2009). Assim, ampliam-se os significados sobre a aprendizagem colaborativa atrelada ao Ensino de Ciências e possibilita-se o pensar sobre o papel dos sujeitos atuando em colaboração.

\section{Tutoria e Mediação no Desenvolvimento Profissional}

Esta categoria aborda o papel do tutor em seus diferentes contextos de atuação. Devido ao processo de interpretação dos trabalhos que foram localizados a partir do seu idioma original de publicação, o inglês, duas nomenclaturas emergiram: o tutor e o mentor. No processo de socialização a seguir adotaremos tutor como única nomenclatura.

Acrescenta-se que uma maneira de aperfeiçoar a compreensão sobre mentoria e a forma de educar seus professores em formação, on-line e presencial, acontece em comunidades de prática (CAUDLE, 2010). O trabalho via e-mail, wikis, mensagens e outros recursos, como fóruns on-line com diálogos escritos em comunidades de prática de formação de mentores e professores constituem a experiência na comunicação mais frequente, com criticidade e a reflexão acerca de suas decisões (SCHERFF; SINGER; BROWN, 2013).

O mentor/tutor é responsável por parte das atividades, ajudando a aliviar a carga de trabalho dos estudantes e dos professores, dá significado ao material pela contextualização, esclarece sobre o enfoque metodológico das atividades ao organizar os assuntos e ao ancorar as discussões (KELLY et al., 2007; TURKICH; GREIVE; COZENS, 2014; SCHERFF; SINGER; BROWN, 2013). Significa-se como o responsável para dar atenção àqueles estudantes ausentes, o sujeito que estreita laços ao organizar grupos, aconseIha acerca do desenvolvimento profissional e de como se comportar em situações de sala de aula, em situações casuais e profissionais (TURKICH; GREIVE; COZENS, 2014). Os comentários dos tutores no formato de áudio encorajam os estudantes a refletirem imediatamente e a agir a partir das demandas de feedback (BROOKES, 2018). É importante ressaltar, contudo, que o encontro síncrono é uma forma de diminuir o anonimato e aumentar a motivação (SCHERFF; SINGER; BROWN, 2013).

O modelo de indagação, associado à tutoria e à tecnologia, abrange o movimento de fazer perguntas e gerar explicações, diálogos críticos e criativos, via compromisso coletivo, de coautoria com os estudantes a partir de interações, negociações e elaborações em grupos (MUUKKONEN-VAN DER MEER, 2011; SWANN, 2013). Tutores podem desenvolver relações amigáveis e empáticas com os estudantes como forma de encora- 
jar, entusiasmar e lhes dar suporte, reconhecendo que os aspectos emocionais da vida dos estudantes são inseparáveis do seu aprendizado e do desenvolvimento de sua confiança profissional (STAFFORD, 2008).

O desenvolvimento profissional de professores ainda não atuantes acontece a partir da experiência como mentores, mostrando-se importante que um colega professor possa contar com o apoio de outro mais experiente (SCHERFF; SINGER; BROWN, 2013). Na formação de tutores os significados do papel da mentoria são negociados quando os tutores engajam-se em ciclos que remodelam suas identidades mediante o questionar, criar hipóteses e compartilhar experiências vividas (CAUDLE, 2010).

Portanto, é preciso a projeção e planejamento de cursos capazes de responder a necessidades emergentes, que incluam diálogo, relação tutor-estudante, e a experiência em um conhecimento cocriado, assim como a possibilidade de ação e interação no feedback (THOMPSON; MACDONALD, 2005). Conclui-se que a mediação do tutor é um fator significativo no desenvolvimento profissional docente, estimulando os processos formativos desse sujeito em ambientes que propiciem a interatividade entre seus participantes.

\section{CONSIDERAÇÕES FINAIS}

Apesar dos diferentes contextos, as produções comunicadas pelos interlocutores (autores das produções acadêmicas) apresentam alguns horizontes para a compreensão dos aspectos teórico-práticos da indagação dialógica na educação. Registra-se que as comunidades on-line na perspectiva do desenvolvimento profissional são mediadas com auxílio de ferramentas e recursos digitais, em um processo de colaboração dos diferentes sujeitos envolvidos, em especial os estudantes, professores e o professor tutor.

Das análises dos estudos localizados nesta revisão emerge a importância dos artefatos tecnológicos na promoção do diálogo e na interação, considerando o papel dos discursos e a apropriação da linguagem científica. No contexto específico do Ensino de Ciências, destacam-se as atividades investigativas colaborativas e o viés dialógico na pesquisa-formação e no desenvolvimento profissional de professores.

Aponta-se para a importância de se desenvolverem ambientes virtuais de aprendizado, alicerçados em uma perspectiva sociocultural, que utilizem recursos tecnológicos na promoção do aprendizado colaborativo, incentivando a cocriação e o aprendizado dialógico. Esses ambientes ainda precisam considerar aspectos socioculturais e envolver os sujeitos em uma postura aprendente de desenvolvimento profissional, além de explorar as diferentes linguagens e recursos, como wikis, fóruns, chats, entre outros.

Reitera-se que o papel do tutor é fundamental e constante em diferentes contextos e modalidades educativas, evidenciado na produção acadêmica como essencial no incentivo ao diálogo, na continuidade da interação com os materiais e as linguagens constituídas pelos diferentes sujeitos, sendo ele próprio parte dos diálogos e fomentador desses. Seu papel é determinante na construção de relações afetuosas com os alunos e professores e na interpretação e auxílio a seus estudantes na compreensão teórica, tecnológica e social, enfatizando a importância de sua presença na formação. 
Por fim, o trabalho evidencia a relevância da indagação dialógica proposta por Gordon Wells na formação de professores para a compreensão dos modos de ser e interagir junto as comunidades colaborativas de desenvolvimento profissional docente. Nesse viés, a produção científica internacional converge em vários aspectos teórico-práticos, como a escrita, leitura e comunicação via ferramentas tecnológicas; a interatividade e a colaboração em redes; o desenvolvimento profissional em comunidades; a aprendizagem colaborativa no Ensino de Ciências e a tutoria e mediação no desenvolvimento profissional. Evidenciou-se aspectos como o diálogo, troca de experiências, partilha de interesses que evoluem socialmente e individualmente no diálogo entre os sujeitos. Apesar dos trabalhos do autor serem em sua maioria comunicados na língua inglesa, os diálogos com seus interlocutores ao longo do trabalho apontam para diferentes contextos de pesquisa, por distintos países e realidades, evidenciando um processo de transformação teórico-prática na formação docente voltada aos diferentes contextos de formação com professores, especialmente atreladas a diferentes formas de comunicação na comunidade, a exemplo daquelas emergentes em cursos de formação na modalidade EaD.

\section{REFERÊNCIAS}

BICUDO, Maria Aparecida Viggiani. Pesquisa qualitativa: segundo a visão fenomenológica. São Paulo: Editora Cortez, 2011.

GALIAZZI, Maria do Carmo et al. Indagações dialógicas com Gordon Wells. 1. ed. Rio Grande: Furg, 2016. p. 47-85.

GUIDOTTI, Charles dos Santos; HECKLER, Valmir. Investigação Dialógica na sala de aula de ciências: etnopesquisa - formação com professores de ciências da natureza. Revista Contexto \& Educação, v. 36, n. 113, p. 143-162, 2021.

KENSKI, Vani Moreira; MEDEIROS, Rosangela Araújo; ORDÉAS, Jean. Ensino Superior em tempos mediados pelas tecnologias digitais. Trabalho \& Educação, v. 28, n. 1, p. 141-152, 2019.

MARTíN-MARTíN, Alberto; THELWALL, Mike; ORDUNA-MALEA, Enrique; LÓPEZ-CÓZAR, Emilio. Google Scholar, Microsoft Academic, Scopus, Dimensions, Web of Science, and OpenCitations' COCl: a multidisciplinary comparison of coverage via citations. Scientometrics, v. 126, n. 1, p. 1-36, 2020.

MORAES, Roque. Reconstruções em interações na linguagem: aprendizagens pela pesquisa em de EAD. Em Teia, Revista de Educação Matemática e Tecnológica Iberoamericana, v. 2, n. 3, p. 1-25, 2011.

PAULA, Adriana Chilante de; HARRES, João Batista. Teoria e Prática no "Educar Pela Pesquisa": Análise de Dissertações em Educação em Ciências. Revista Contexto \& Educação, v. 30, n. 96, p. 156-192, 2016.

VERGARA, Sylvia Constant; PECl, Alketa. Escolhas metodológicas em estudos organizacionais. Organizações \& Sociedade, v. 10, p. 13-26, 2003.

WELLS, Gordon. Action, talk, and text: Learning and teaching through inquiry. Nova York: Teachers College Press, 2001.

WELLS, Gordon. Dialogic inquiry: Towards a socio-cultural practice and theory of education. Cambrige: Cambridge University Press, 1999.

WELLS, Gordon. Inquiry as an Orientation for Learning, Teaching and Teacher Education. In: WELLS, Gordon; CLAXTON, Guy. Learning for Life in the 21st Century: Sociocultural Perspectives on the Future of Education. Oxford: Blackwell Publishers, 2002, p. 197-210.

\section{APÊNDICE: Listagem das produções analisadas}

AKTEKIN, Nafiye Çiğdem. The impact of in-service teacher education (INSET) programme on professional development of EFL teachers through the critical friends group (CFG). 2013. Tese (Doutorado) - Cukurova University, Adana, 2013. 
ANSON, Joseph P. Writing Attitudes and Practices of Content Area Teachers after Participating in the Central Utah Writing Project Summer Institute. 2017. Tese (doutorado) - Utah State University, Logan, 2017.

AWENOWICZ, Melissa Anne. The influence of beliefs and cultural models on teacher candidates' professional identities and practices. 2009. Tese (Doutorado) - University of Pittsburgh, Pittsburgh, 2009.

BAINBRIDGE, Jonathan; COUNSELL, Karen; GREALY, Freda; MAHARG, Paul; MILLS, Joel; O'BOYLE, Rory. iPads in Legal Learning (iLEGALL): mobile devices in professional legal learning. European Journal of Law and Technology, v. 4, n. 1, 2013.

BAKER, Will; WATSON, Julie. Mastering the online master's: Developing and delivering an online MA in English language teaching through a dialogic-based framework. Innovations in Education and Teaching International, v. 51, n. 5, p. 483-496, 2014.

BARAB, Sasha A.; EVANS, Michael A.; BAEK, Eun-Ok. Activity theory as a lens for characterizing the participatory unit. In: Handbook of research on educational communications and technology. Routledge, 2013. p. 208-223.

BARTLETT-BRAGG, Anne. An investigation into adult learners' experiences of developing distributed learning networks with self-publishing technologies. 2013. Tese (Doutorado) - University of Technology, Sydney, 2013.

BERNASCONI, Natalie. Teacher collaborative leadership on behalf of access to technological literacy for English Language Learners. 2010. Tese (Doutorado) - University of California, Santa Cruz, 2010.

BERRY, Stuart. Tacit knowing made visible: The use and value of an online archive. 2014. Tese (Doutorado) - Athabasca University, Athabasca, 2014.

BEZAIRE, Kimberly. When it's choosing time: Boys' multiliteracies at play. 2009. Tese (Doutorado) - University of Toronto, Toronto, 2009.

BROOKES, Alexandra Alana. The Dialogic Potential of Providing Audio Versus Written Assignment Feedback in Higher Education: A Mixed Method Study. 2018. Dissertação (Mestrado) - Aberystwyth University, Aberystwyth, 2018.

BRUCE, David; SULLIVAN, Sunshine. Writing with Video: Learning and Sharing in Communities of Practice. The English Record, v. 68, n. 2, p. 1-22, 2018.

BUNCH, George C. Pedagogical language knowledge: Preparing mainstream teachers for English learners in the new standards era. Review of research in education, v. 37, n. 1, p. 298-341, 2013.

CARON, André H.; CARONIA, Letizia. The cultural dimensions of the adoption of "Ipods" in higher education. IADIS International Journal on WWW/Internet, v. 6, n. 2, p. 73-89, 2008.

CAUDLE, Lori Allison. The Professional Development of Pre-K Mentor Teachers: Insights from a Face-to-Face and Online Community of Practice. 2010. Tese (Doutorado) - University of Tennessee, Tennessee, 2010.

CHAl, Ching Sing. Teachers' professional development in a computer-supported collaborative learning environment: A descriptive and interpretive enquiry. 2006. Tese (Doutorado) - University of Leicester, United Kingdom, 2006.

CHAl, Ching Sing; TAN, Seng Chee. Computer-Supported collaborative learning for knowledge creation. In: FISHER, Darrell L. KHINE, Myint Swe. Contemporary Approaches To Research On Learning Environments: Worldviews. Singapura: World Scientific Publishing, 2006. p. 579-601.

CHEN, Chao-hsiu. Investigating the influences of teacher belief and contextual factors on the technology integration of Taiwanese high school teachers. 2006. Tese (Doutorado) - University of Texas, Austin, 2006.

CHILDS, Sharon S. Language teacher cognition: Tracing the conceptualizations of second language teachers. 2011. Tese (Doutorado) - Pennsylvania State University, Pennsylvania, 2011.

CHRISTIANSEN, Catey. Creating classroom communities of practice: Students as practioners of content. 2010. Dissertação (Mestrado) - The Evergreen State College, Olympia, 2010.

CLARKE, Lisa. Improving Principal Professional Practice Through Communities of Practice. 2017. Tese (Doutorado) - Western University, Ontario, 2017.

CLARKE, Matthew. Language teacher identities. Clevedon: Multilingual Matters, 2008.

COONER, Tarsem Singh. Creating opportunities for students in large cohorts to reflect in and on practice: Lessons learnt from a formative evaluation of students' experiences of a technology-enhanced blended learning design. British Journal of Educational Technology, v. 41, n. 2, p. 271-286, 2010. 
COPPERSMITH, Sarah A. Teacher Candidates in Context: Investigating Inquiry Learning, Self-Efficacy and Stress within Teacher Education. International Online Journal of Teachers in Collaboration, v. 1, n. 2, p. 13-39, 2017.

DESTINO, Thomas. Observing a bilingual science teacher accommodating both language and content objectives. 1994. Tese (Doutorado) - Ohio State University, Ohio, 1994.

DONNELLY, Roisin. The nexus of problem-based learning and learning technology: Does it enable transformative practice? 2009. European Journal of Open, Distance and E-Learning, 2009. Disponível em: http:// www.eurodl.org/?p=current\&article=371. Acesso em: 30 jun. 2021.

DOYLE, Kieran. Evaluating and improving the delivery of work-based learning. 2014. Tese (Doutorado) Dublin City University, Dublin, 2014.

$\mathrm{ENOCH}$, Jerol. Learning language with technology in a hybrid university EAP course. 2016. Tese (Doutorado) - University of Missouri, Saint Louis, 2016.

FAHLE, Kimberly. Collaboration and Community in Undergraduate Writing Synchronous Video Courses (SVCS). 2019. Tese (Doutorado) - Old Dominion University, Norfolk, 2019.

FARMER, James; BARTLETT-BRAGG, Anne. Blogs@ anywhere: High fidelity online communication. In: ASCILITE 2005: Balance, Fidelity, Mobility: Maintaining The Momentum? 2005, Brisbane. Proceeding [...]. 2005. p. 197-203.

FAROOQ, Sana. The Critically Reflective Practice of Online Educators: Constructing a Dialogic Pedagogy in Virtual Learning Environments. 2019. Dissertação (Mestrado) - Auckland University of Technology, Auckland, 2019.

FARRELL, Robert; BADKE, William. Situating information literacy in the disciplines: A practical and systematic approach for academic librarians. Reference Services Review, v. 43, n. 2, p. 319-340, 2015.

FLYNN, Theresa; POLIN, Linda. Making Sense of Online Learning: Frames, Rubrics, Tools \& Coding Systems for Analyzing Asynchronous Online Discourse. In: AERA 2003, Artigo, Chicago, 2003, p. 1-28.

GAUDIN, Cyrille; CHALIÈS, Sébastien. Video viewing in teacher education and professional development: A literature review. Educational Research Review, v. 16, p. 41-67, 2015.

GELER, Chelsea; NEISLER, David. Two future Teachers' views on Peer-Supported Learning. In: GARCIA, Antero. Teaching in the Connected Learning Classroom. Invine: Digital Media and Learning Research Hub., 2014. p. 32-38.

GONZÁLEZ, Jorge García. Innovación educativa en las redes sociales online: un estudio en la educación superior. 2015. Tese (Doutorado) - Universidad Politécnica de Madrid, Madrid, 2015.

GRAETZ, Catherine M. Mutual benefits? Investigating a service teaching learning partnership. 2008. Tese (Doutorado) - University of the Witwatersrand, Johannesburg, 2007.

HADDOCK, Lucy. A Comparison Of Teachers' Beliefs Of The Use Of Inquiry Teaching, Origin Of Knowledge Of Inquiry Teaching, And Student Achievement Between International Baccalaureate And Non-International Baccalaureate Primary Years Programme Schools. 2014. Tese (Doutorado) - University of Central Florida, Florida, 2014.

HAN, Ninghua. The effect of discussion protocol and modeling example on students' participation, quality, and perceptions of asynchronous online discussion. 2013. Tese (Doutorado) - Texas Tech University, Lubbock, 2013.

HAUGHT, John Richard. Embodied language performance: Mediational affordances of dramatic activity for second language learning. 2005. Tese (Doutorado) - University of Nevada, Las Vegas, 2005.

HELLERMANN, John. Social actions for classroom language learning: New Perspectives on Language and Education. Clevedon: Multilingual Matters, 2008.

HENNESSY, Sara; LONDON, Laura. Learning from international experiences with interactive whiteboards: The role of professional development in integrating the technology. OECD Education Working Papers, $\mathrm{n}$. 89, p. 4-25, 2013.

HENNESSY, Sara; MERCER, Neil; WARWICK, Paul. A dialogic inquiry approach to working with teachers in developing classroom dialogue. Teachers College Record, v. 113, n. 9, p. 1.906-1.959, 2011.

HILL, Janette R.; WILEY, David; NELSON, Laurie Miller.; HAN, Seungyeon. Exploring Research on Internet-based Learning: From Infrastructure to Interactions In: JONASSEN, David H. Handbook of Research on Educational Communications and Technology. 2. ed. Mahwah: Lawrence Erlbaum Associates, 2008. p. 433-460. 
HILL, LeRoy. A learning design approach for exploring a framework for mediating collaborative knowledge-building in the Caribbean Educators Network. 2011. Tese (Doutorado) - University of Nottingham, Nottingham, 2011.

HOANG, Ngoc Tue. EFL teachers' perceptions and experiences of blended learning in a Vietnamese university. 2015. Tese (Doutorado) - Queensland University of Technology, Queensland, 2015.

HUANG, Chung-Kai. Learner satisfaction with blog-and wiki-supported writing in an EFL course in Taiwan. 2012. Tese (Doutorado) - University of Texas, Austin, 2012.

HUNG, Hsiu-Ting. A Reflective Practitioner Approach to Teacher Preparation: Learning in and through Communities of Practice. 2005. Tese (Doutorado) - University of Georgia, Athens, 2005.

JAMIL, Md Golam. Technology enhanced teacher-learning in rural Bangladesh: a critical realist inquiry with secondary teachers of English. 2015. Tese (Doutorado) - University of Southampton, Southampton, 2015.

JAWORSKI, Barbara. Mathematics Education Development Research in Teaching. Learning in Practice. In: 37th ANNUAL CONFERENCE OF THE MATHEMATICS EDUCATION RESEARCH GROUP OF AUSTRALASIA. Proceedings, Sydney, 2014, p. 2-23.

JAWORSKI, Barbara. Research practice into/influencing mathematics teaching and learning development: Towards a theoretical framework based on co-learning partnerships. Educational Studies in Mathematics, v. 54, n. 2, p. 249-282, 2003.

JEWITT, Katharine Elizabeth. Using virtual reality to enhance informal learning in small and medium enterprises. 2018. Tese (Doutorado) - University of Glasgow, Glasgow, 2018.

KARLSSON, Göran. Learning Science by Digital Technology. Students' understanding of computer animated learning material. 2010. Trabalho (Conclusão de Curso) - Chalmers University of Technology \& University of Gothenburg, Gothenburg, 2010.

KELLY, Nick. Online networks in teacher education. In: ALLEN, Tim. Oxford Research Encyclopedia of Education, v. 6, n. 42, p. 1-21, 2019.

KELLY, Nick; CLARÀ, Marc; KEHRWALD, Benjamin; DANAHER, Patrick Alan. Online Learning Networks for Pre-service and Early Career Teachers. Londres: Macmillan Publishers, 2016.

KELLY, Peter; GALE, Ken; WHEELER Steve; TUCKER, Viv. Taking a stance: promoting deliberate action through online postgraduate professional development. Technology, Pedagogy and Education, v. 16, n. 2, p. 153-176, 2007.

KHOO, Elaine G. L. Developing an online learning community: A strategy for improving lecturer and student learning experiences. 2010. Tese (Doutorado) - University of Waikato, Hamilton, 2009.

KHOUSA, Eman Abu; ATIF, Yacine; MASUD, Mohammad M. A social learning analytics approach to cognitive apprenticeship. Smart Learning Environments, v. 2, n. 1, p. 1-23, 2015.

KLENNER-MOORE, Jayne. Dialogic Enquiry in an Online Community. In: E-Learn: World Conference on E-Learning in Corporate, Government, Healthcare, and Higher Education. Association for the Advancement of Computing in Education (AACE), Conference Paper. San Diego, 2002. p. 1.714-1.717.

KOKKOTAS, Panagiotis; PILIOURAS, Panagiotis. Bridging history of science and science education: "The MAP project". In: IHPST 2005, Artigo, 2005. p. 1-10.

KOKKOTAS, Panos; PILIOURAS, Panagiotis; MALAMITSA, Katerina; STAMOULIS, Efthymios. Teaching physics to in-service primary school teachers in the context of the history of science: the case of falling bodies. Science \& Education, v. 18, n. 5, p. 609-629, 2009.

KOVALAINEN, Minna. The social construction of learning and teaching in a classroom community of inquiry. 2013. Tese (Doutorado) - University of Oulu Graduate School, Finland, 2013.

LEE, Cheun-Yeong. A Case Study of Using Synchronous Computer-Mediated Communication System for Spoken English Teaching and Learning Based on Sociocultural Theory and Communicative Language Teaching Approach Curriculum. 2009. Tese (Doutorado) - Ohio University, Ohio, 2009.

LEE, Sangmin. Electronic spaces as an alternative to traditional classroom discussion and writing in secondary English classrooms. Journal of Asynchronous Learning Network, v. 9, n. 3, p. 25-46, 2005.

LINDA, Polin. Learning in Dialogue with a Practicing Community. In: DUFFY, Thomas M.; KIRKLEY, Jamie R. Learner Centerede Theory and Practice in Distance Education: Cases From Higher Education. Mahwah: Lawrence Erlbaum Associates, 2004. p. 17-48.

LOCKHORST, Ditte. Design principles for a CSCL environment in teacher training. Utrecht: IVLOS, 2004. 
LUND, Andreas; HAUGE, Trond Eiliv. Designs for teaching and learning in technology-rich learning environments. Nordic Journal of Digital Literacy, v. 6, n. 4, p. 258-272, 2011.

MAGGIOLI, Gabriel Diaz. Understanding Expert Mediation in Online and On-site Settings: A Case Study. 2018. Tese (Doutorado) - University of Bath, Bath, 2018.

MARDEN, Mariolina Pais. Investigating an online community of second language learners using design-based research. 2016. Tese (Doutorado) - Murdoch University, Perth, 2016.

MATTHEW, Kathryn I.; FELVEGI, Emese; CALLAWAY, Rebecca A. Wiki as a collaborative learning tool in a language arts methods class. Journal of Research on Technology in Education, v. 42, n. 1, p. 51-72, 2014.

MCPHEE, Eleanor Anee. Shared concerns: Investigating ways instrumental teachers learn to teach. 2013. Tese (Doutorado) - University of Western Sydney, Sydney, 2013.

MERI-YILAN, Serpil. Take your time'to "find yourself!": an exploration of scaffolded autonomous e-learning environments amongst international students in a UK university. 2017. Tese (Doutorado) - University of Southampton, Southampton, 2017.

MLITWA, Nhlanhla BW. Integration and use of educational technology in undergraduate curicular at the University of Zululand - An ACTAD perspective. In: INTERNATIONAL CONFERENCE ON EDUCATION AND NEW LEARNING TECHNOLOGIES, 9., 2017. Barcelona. Proceeding. Barcelona, 2017. p. 3-5.

MOREIRA, J. António; ALMEIDA, Ana Cristina. How reliable and consistent is our learning community of inquiry? Psychometric qualities of the community inquiry survey instrument applied to a sample of higher education portuguese students. In: INTERNACIONAL CONFERENCE ON EDUCATION AND NEW LEARNING TECHNOLOGIES (EDULEARN). International Association of Technology, Education and Development (IATED), 2011. p. 4.261-4.266.

MORGAN, Anne-Marie. Online collaborative communities of learning for pre-service teachers of languages. Babel, v. 50, n. 2, p. 36-43, 2015.

MUUKKONEN-VAN DER MEER, Hanni et al. Perspectives on knowledge creating inquiry in higher education. 2011. Trabalho (Conclusão de Curso) - University of Helsinki, Finland, 2011.

NELSON, Tamara Holmlund. Building leadership capacity by nurturing community. In: WIESEMAN, Katherine C.; WEINBURGH, Molly H. Women's Experiences in Leadership in K-16 Science Education Communities. Dordrecht: Springer, 2009. p. 167-181.

PADILLA, Lindsay. "My gut has to feel it": A participatory action research study of community college educators navigating the emotional terrain of human rights education. 2014. Tese (Doutorado) - University of San Francisco, San Francisco, 2014.

PÁEZ, Patricia Thibaut. Literacy and Learning across Physical and Digital Spaces: A Case Study in a Blended Primary Classroom. 2015. Tese (Doutorado) - University of Sydney, Sydney, 2015.

PILIOURAS, Panagiotis; PLAKITSI, Katerina; NASIS, Georgios. Discourse Analysis of Science Teachers Talk as a Self-Reflective Tool for Promoting Effective NOS Teaching. World Journal of Education, v. 5, n. 6, p. 96-107, 2015.

PLAKITSI, Katerina; PILIOURAS, Panagiotis; EFTHIMIOU, George. Discourse analysis: a tool for helping educators to teach science. Forum Qualitative Sozialforschung/Forum: Qualitative Social Research, v. 18, n. 1, p. 28, 2017.

PRITCHARD, Malcolm Ronald. Experiential learning programs in Australian secondary schools. 2010. Tese (Doutorado) - University of Melbourne, Melbourne, 2010.

QHOBELA, Makomosela. Construction of meaning by second language students in access physics classes in Lesotho. 2008. Tese (Doutorado) - University of the Witwatersrand, Johannesburg, 2008.

QUIÑONES QUIÑONES, Katerine Maribel et al. Promoviendo aspectos de la conciencia intercultural al incorporar elementos de la danza. 2017. Dissertação (Mestrado) - Universidad Distrital Francisco José de Caldas, Bogotá, 2017.

RESCHKE, Kathy Lynn. Knowledge building among family child care providers during a training using problem-based learning and computer-mediated communication. 2001. Tese (Doutorado) - lowa State University, Ames, 2001.

REZEKI, Yanti Sri. Indonesian english-as-A-foreign-language (EFL) learners' experiences in collaborative writing. 2016. Tese (Doutorado) - University of Rochester, Rochester, 2016. 
ROBUTTI, Ornella; CUSI, Annalisa. CLARK-WILSON, Alison; JAWORSKI, Barbara; CHAPMAN, Olive; ESTELEYS, Cristina; GOOS Merrilyn; ISODA Masami; JOUBERT, Marie. ICME international survey on teachers working and learning through collaboration: June 2016. ZDM Mathematics Education, v. 48, n. 5, p. 651690, 2016.

ROWELL, Patricia M. Learning in school science: The promises and practices of writing. Studies in Science Education, v. 30, n. 2, p. 19-56, 2008.

SARITAS, Mustafa Tuncay. Computer-mediated communication in higher education: An exploration of knowledge construction. 2006. Tese (Doutorado) - lowa State University, Ames, 2006.

SCHERFF, Lisa; SINGER, Nancy Robb; BROWN, Alan. Mentoring "Pre” Preservice Teachers in Third Spaces. Teacher Education and Practice, v. 26, n. 3, 2013.

SEELS, Barbara; FULLERTON, Karen; BERRY, Louis; HORN. Laura J. Research on Learning from Television. In: JONASSEN, David H. Handbook of Research on Educational Communications and Technology. 2. ed. Mahwah: Lawrence Erlbaum Associates, 2004. p. 249-334.

SHEEHAN, Dale; HIGGS, Joy. Practice-based education: theoretical underpinnings. In: HIGGS, Joy; SHEEHAN, Dale; CURRENS, Julie Baldry; LETTS, Will; JENSEN, Gail M. Realising Exemplary Practice-Based Education. Rotterdam: Sense Publishers, 2013. p. 13-24.

SHUKOR, Siti Shuhaida. Effectiveness of face-to-face and Facebook collaborative writing in Malaysian ESL undergraduates' writing performance. 2014. Dissertação (Mestrado) - Universiti Putra Malaysia, Serdang, 2014.

SIGALA, Marianna. Integrating Web 2.0 in e-learning environments: a socio-technical approach. International journal of knowledge and learning, v. 3, n. 6, p. 628-648, 2007.

SIMPSON, Alyson. The use of Children's Literature in Teaching: A study of politics and professionalism with teacher education. New York: Routledge, 2016.

SINCLAIR, Christine Moira. Students and discourse: An insider perspective. 2004. Tese (Doutorado) - Open University, United Kingdom, 2004.

SLAVIT, David; NELSON, Tamara Holmlund; LESSEIG, Kristin. The teachers' role in developing, opening, and nurturing an inclusive STEM-focused school. International Journal of STEM Education, v. 3, n. 1, p. 7, 2016. SPILIOTOPOULOS, Valia; CAREY, Stephen. Investigating the role of identity in writing using electronic bulletin boards. Canadian modern language review, v. 62, n. 1, p. 87-109, 2016.

STAFFORD, Patricia Margaret. Practising what we preach: a study of pedagogical interactions in the context of teacher education. 2008. Tese (Doutorado) - University of Leicester, United Kingdom, 2008.

STEELE, Haley Kay. Identity and motivation for engagement within a professional distributed community of practice. 2009. Tese (Doutorado) - University of Texas, Austin, 2009.

STONE, Rachael Anne. A Study of Primary School Teachers' Understandings and Perceptions of Teaching Reading. 2018. Tese (Doutorado) - Canterbury Christ Church University, Canterbury, 2018.

SUBRAMANIAM, Karthigeyan. Constructing classroom meaning with the integration of computer technology into teaching. 2001. Tese (Doutorado) - University of Otago, Dunedin, 2001.

SWANN, Jennie. Dialogic inquiry: from theory to practice. 2013. Tese (Doutorado) - University of Southern Queensland, Queensland, 2013.

TAN, Seng-Chee. Technologies for adult and lifelong education. In: MILANA, Marcela; WEBB, Sue; HOLFORD, John; WALLER, Richard; JARVIS, Peter. The palgrave international handbook on adult and lifelong education and learning. Palgrave Macmillan, London, 2018. p. 917-937.

TAN, Seng-Chee. Learning with computers: Generating insights into the development of cognitive tools using cultural historical activity theory. Australasian Journal of Educational Technology, v. 35, n. 2, 2019.

TAPP, Jane. Being and becoming a student: an investigation into how a pedagogic approach built on collaborative participation in academic literacy practices supports students' academic practice, knowlege and identity. 2013. Tese (Doutorado) - University of Nottingham, Nottingham, 2013.

THOMPSON, Terrie Lynn. The Virtual Classroom @ Work. In: ZEMLIANSKY, Pavel; ST. AMANT, Kirk. Handbook of Research on Virtual Workplaces and the New Nature of Business Practices. New York: Informatlon sclence reference, 2008. p. 272288.

THOMPSON, Terrie Lynn; MACDONALD, Colla J. Community building, emergent design and expecting the unexpected: Creating a quality eLearning experience. The Internet and Higher Education, v. 8, n. 3, p. 233249, 2005. 
TROIANO, Beverly. Developing professional teacher researchers: Transforming language learning through discourse analysis. 2012. Tese (Doutorado) - University of Illinois, Illinois, 2012.

TURKICH, Kent; GREIVE, Shane; COZENS, Paul. Transferring educational theories and knowledge using a co-teaching mentor model: A discipline-appropriate approach. Journal of University Teaching and Learning Practice, v. 11, n. 3, 2014.

TWINING, Peter; BROADIE, Roger; COOK, Deirdre; FORD, Karen; MORRIS, DAVID; TWINER, Alison; UNDERWOOD, Jean. Educational change and ICT: an exploration of priorities $2 \& 3$ of the DfES eStrategy in schools and colleges. Coventry: Becta, 2016.

VERJOVSKY, Janet; WALDEGG, Guillermina. Analyzing beliefs and practices of a Mexican high school biology teacher. Journal of Research in Science Teaching: The Official Journal of the National Association for Research in Science Teaching, v. 42, n. 4, p. 465-491, 2005.

VICKERS-MANZIN, Jen; JOHNSTON, Jan. A Living Educational Theory of Knowledge Translation: Improving Practice, Influencing Learners, and Contributing to the Professional Knowledge Base. 2013. Tese (Doutorado) - Brock University, St Catharines, 2013.

WELLS, Gordon. Aprendizagem Dialógica: o Processo dos Seres Humanos de Falar em Direção à Compeensão. In: GALIAZZI, Maria do Carmo. et al. Indagações dialógicas com Gordon Wells. 1. ed. Rio Grande: Editora da FURG, 2016. p. 47-85.

WESTBERRY, Nicola Catherine. An activity theory analysis of social epistemologies within tertiary-level eLearning environments. 2009. Tese (Doutorado) - University of Waikato, Hamilton, 2009.

WILMES, Sara. Student-driven inquiry-based science education in Luxembourg primary school contexts. 2017. Tese (Doutorado) - University of Luxembourg, Esch-sur-Alzette, 2017.

YEN, Ai Chun. Critical Reading and Writing: A Literary Project for Non-English Majors with Thinking Tools. Journal of Educational Technology Development and Exchange (JETDE), v. 4, n. 1, p. 5, 2011.

YIM, Yoon-kyung Kecia. Second language students' discourse socialization in academic online communities. Canadian Modern Language Review, v. 67, n. 1, p. 1-27, 2011.

YUKAWA, Joyce. Hearts and minds through hands online: a narrative analysis of learning through co-reflection in an online action research course. 2005. Tese (Doutorado) University of Hawai'i, Manoa, 2005.

ZULU, Sphamandla Innocent. Exploring Grade 10 rural physical science teachers' perceptions and usage of everyday words in Acornhoek science classrooms, Mpumalanga Province. 2018. Dissertação (Mestrado) University of the Witwatersrand, Johannesburg, 2018. 